\title{
In vitro comparison of aortic valve movement after valve-preserving aortic replacement
}

Roland Fries, MD, ${ }^{\text {a }}$ Thomas Graeter, MD, ${ }^{\mathrm{b}}$ Diana Aicher, MD, ${ }^{\mathrm{b}}$ Helmut Reul, MD, ${ }^{\mathrm{c}}$ Christoph Schmitz, ${ }^{\mathrm{c}}$ Michael Böhm, MD, and Hans-Joachim Schäfers, $M^{b}$

From the Departments of Cardiology a and Thoracic and Cardiovascular Surgery, ${ }^{\mathrm{b}}$ University Hospital Homburg, Homburg/Saar, Germany, and the Helmholtz Institute for Biomedical Engineering, ${ }^{\mathrm{C}}$ University of Technology Aachen, Germany.

Received for publication Nov 29, 2005; revisions received Jan 11, 2006; accepted for publication Feb 6, 2006.

Address for reprints: Roland Fries, GotthardSchettler-Klinik, Prof.-Kurt-Sauer-Str. 4, 76669 Bad Schönborn, Germany (E-mail: r.fries@gotthard-schettler-klinik.de).

J Thorac Cardiovasc Surg 2006;132:32-7

$0022-5223 / \$ 32.00$

Copyright (๑) 2006 by The American Association for Thoracic Surgery

doi:10.1016/j.jtcvs.2006.02.034
Objective: In aortic valve regurgitation and aortic dilatation, preservation of the aortic valve is possible by means of root remodeling (Yacoub procedure) or valve reimplantation (David procedure). In vivo studies suggest that reimplantation might substantially influence aortic valve-motion characteristics. Evaluation of aortic valve movement in vivo, however, is technically limited and is difficult to standardize. We evaluated the aortic valve-motion pattern echocardiographically in vitro after reimplantation and remodeling.

Methods: By using aortic roots of house pigs (aortoventricular diameter, $22 \mathrm{~mm}$ ) a Yacoub procedure (22-mm graft; Yacoub, $\mathrm{n}=5$ ) or a David I procedure (24-mm graft; David, $\mathrm{n}=5$ ) was performed. Roots after supracommissural replacement (22-mm graft; Supra, $\mathrm{n}=5$ ) served as control valves. In an electrohydraulic, computer-controlled pulse duplicator the valves were tested at flows of 2, 4, 7, and 9 L/min. Echocardiographically assessed parameters were rapid valve-opening velocity, slow valve-closing velocity, rapid valve-closing velocity, rapid valveopening time, rapid valve-closing time, ejection time, maximum valve opening, slow valve-closing displacement, and maximum flow velocity.

Results: Mean rapid valve-opening velocity and mean rapid valve-closing velocity at a cardiac output of 2 to $9 \mathrm{~L} / \mathrm{min}$ were fastest in David (rapid valve-opening velocity: $69 \pm 10 \mathrm{~cm} / \mathrm{s}$ [David] vs $39 \pm 4 \mathrm{~cm} / \mathrm{s}$ [Yacoub] vs $42 \pm 4 \mathrm{~cm} / \mathrm{s}$ [Supra], $P=$ .0041 ; rapid valve-closing velocity: $22 \pm 2 \mathrm{~cm} / \mathrm{s}$ [David] vs $16 \pm 2 \mathrm{~cm} / \mathrm{s}$ [Yacoub] vs $17 \pm 1 \mathrm{~cm} / \mathrm{s}$ [Supra], $P=.0272)$, and slow valve-closing velocity was slowest in David $(0.2 \pm 0.1 \mathrm{~cm} / \mathrm{s}$ [David] vs $1.0 \pm 0.3 \mathrm{~cm} / \mathrm{s}$ [Yacoub] vs $0.6 \pm 0.1 \mathrm{~cm} / \mathrm{s}$ [Supra], $P=.0063)$. With increasing cardiac output, the difference in rapid valve-opening velocity between the groups increased, the difference in slow valveclosing velocity remained unchanged, and the difference in rapid valve-closing velocity decreased.

Conclusions: In this standardized experimental setting remodeling of the aortic valve provides significantly smoother valve movements. This might contribute to preservation of a better valve performance during long-term follow-up.

$\mathrm{V}$ alve-preserving operations for the treatment of valve regurgitation and aortic root dilatation are of increasing clinical interest. Feasibility with low perioperative morbidity and mortality ${ }^{1-4}$ and convincing functional results during midterm follow-up have been proved in several series. ${ }^{5-8}$ The 2 dominating techniques are the remodeling technique introduced by Sarsam and Yacoub ${ }^{1}$ and the reimplantation technique proposed by David, ${ }^{2,3}$ both using a Dacron tube for replacement of the diseased aortic wall. In contrast to the Yacoub procedure, the original David procedure (David I) does not include restoration of the neosinuses.

Aortic valve function involves a complex interplay of the aortic root and cusps during the cardiac cycle. ${ }^{9-11}$ Valve-preserving aortic replacement might alter the 


\section{Abbreviations and Acronyms \\ ET = ejection time \\ $\mathrm{RVCV}=$ rapid valve-closing velocity \\ $\mathrm{RVOV}=$ rapid valve-opening velocity \\ $\mathrm{SVCV}=$ slow valve-closing velocity}

functional properties of the aortic valve by changing the geometry, elasticity, and mobility of the anatomic structures. The opening and closing characteristics of the aortic valve might have implications on its durability and have been repeatedly studied in vivo. ${ }^{13-16}$ Different movement patterns of the aortic valve after remodeling and reimplantation have been described, suggesting that aortic valve movement might be more physiologic after valve remodeling.

Evaluation of aortic valve movement by means of echocardiography in vivo, however, is limited by the lack of standardized hemodynamic and anatomic conditions. Differences in blood pressure, cardiac output, and heart rate during the examination might significantly influence echocardiographic measurements. Furthermore, the echocardiographic view on the aortic cusps, which determines the results of M-mode measurements, varies considerably according to the individual anatomy of each patient. The purpose of this study was to evaluate echocardiographically the opening and closing characteristics of the aortic valve after remodeling or reimplantation. To generate standardized anatomic and hemodynamic conditions, an in vitro model using an electrohydraulic pulse duplicator was used.

\section{Methods}

\section{Study Objects}

Fresh porcine hearts including the ascending aorta were obtained from a local slaughterhouse. Only tricuspid aortic valves with an aortoventricular diameter of $22 \mathrm{~mm}$ were used for this study. The aortoventricular junction was carefully prepared, removing the entire left ventricle except for a $1-\mathrm{cm}$ rim below the aortic valve insertion line. A 24-mm vascular prosthesis was connected to the remnants of the left ventricular outflow tract.

In 5 aortic roots a remodeling procedure was carried out using a standard technique. ${ }^{1}$ The aortic sinuses were excised, and a 22-mm Dacron graft was configured so that the edges resembled the insertion lines of the aortic cusps. The graft was then sutured to the aortic valve insertion, thus remodeling the aortic root. The reimplantation technique was applied to 5 porcine aortic roots. The aortic sinuses were excised, and a 24-mm Dacron graft was anchored to the aortoventricular junction. The aortic valve was then reimplanted within the graft in typical fashion. ${ }^{2}$ As a control group, we used 5 aortic roots with supracommissural replacement with a 22-mm Dacron graft.

\section{Study Protocol}

The design of the pulse duplicator (Figure 1) was based on experimental studies of Reul and colleagues. ${ }^{17,18}$ In this simulated

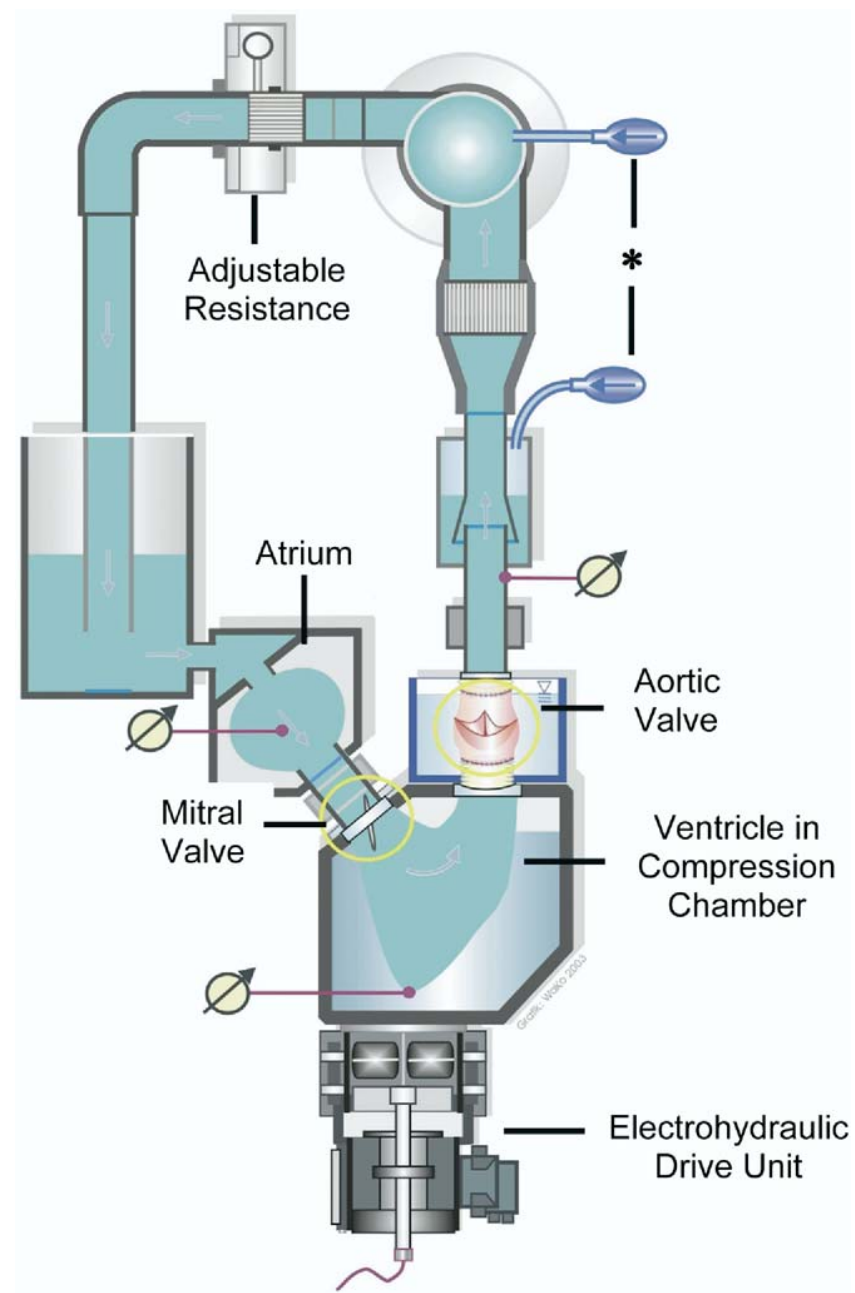

Figure 1. Schematic of the pulse duplicator. *Adjustable compliances.

circulation blood pressure, heart rate, and cardiac output can be chosen to mimic physiologic conditions at rest and during exercise. The circulation contains a silicon sac simulating the left ventricle and a mechanical silicon valve, which is placed in the mitral position. Afterload can be adjusted manually to maintain standardized blood pressure.

In aortic valve position we inserted the aortic root preparations, connecting the prosthesis below the valve and the ascending aortic prosthesis to the circulation. The testing conditions were set in accordance with the international US Food and Drug Administration interlaboratory comparison protocol, ${ }^{19}$ working at a fixed heart rate of 70 beats/min. Mean aortic pressure was $100 \mathrm{~mm} \mathrm{Hg}$, and the cardiac outputs were increased from 2 up to 4,7 , and 9 $\mathrm{L} / \mathrm{min}$. Saline $(0.9 \%)$ was used as fluid.

\section{Echocardiography}

Aortic roots were positioned in a Perspex chamber filled with saline. To obtain optimal echocardiographic recordings, the width 


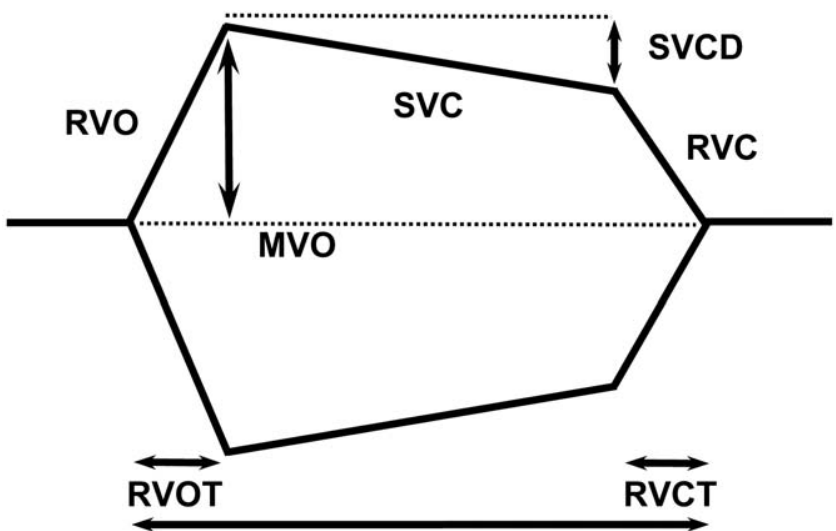

ET

Figure 2. Schematic of the valve-motion pattern. RVO, Rapid valve opening; SVC, slow valve closing; SVCD, slow valve-closing displacement; $R V C$, rapid valve closing; $M V O$, maximum valve opening; RVOT, rapid valve-opening time; $R V C T$, rapid valve-closing time; $E T$, ejection time.

of this chamber was constructed so that the ultrasound transducer could be placed closely, without the aortic root touching the chamber wall. Echocardiograms were performed on a Philips HDI 5000 system with a $12.5 \mathrm{MHz}, 38-\mathrm{mm}$ linear array ultrasound transducer. The noncoronary sinus of the aortic valve was placed nearest to the ultrasound transducer, so that the noncoronary aortic valve cusp opened and closed precisely within the ultrasound axis in each aortic root. M-mode tracings were recorded at $100 \mathrm{~mm} / \mathrm{s}$ on videotapes, choosing maximum magnification. Still frames of these tracings were analyzed subsequently offline by using the system internal software. Valve opening and closing was characterized according to the protocol of Leyh and associates, ${ }^{13}$ including the following parameters: rapid valve-opening velocity (RVOV) and rapid valve-closing velocity (RVCV), rapid valve-opening time and rapid valve-closing time, slow valve-closing velocity (SVCV), ejection time (ET), maximum valve opening, and leaflet displacement during slow leaflet closing (Figure 2). Maximum flow velocities across the aortic valve were obtained by means of pulsedwave Doppler scanning. The Doppler volume was placed just in front of the aortic valve cusps in the middle of the aortic root. For each parameter, mean values were calculated from 3 cardiac cycles.

\section{Statistical Analysis}

Results were expressed as means \pm standard error. A 2-way analysis of variance was used for comparison of continuous parameters among the 3 groups. Statistical significance was established at a $P$ value of less than .05. Statistical analysis was performed with StatView software (version 4.0) for the Macintosh.

\section{Results}

M-mode measurements revealed significantly rougher valve movements in reimplanted valves with respect to the mean values of the calculated valve-motion parameters at cardiac
TABLE 1. Valve-motion characteristics after reimplantation (David) and remodeling (Yacoub) and in control valves (Supra): Pooled mean values at cardiac outputs of 2, 4, 7, and $9 \mathrm{~L} / \mathrm{min}$

\begin{tabular}{lcccc}
\hline & David & Yacoub & Supra & $P$ value \\
\hline RVOV (cm/s) & $69 \pm 10$ & $39 \pm 4$ & $42 \pm 4$ & .0041 \\
SVCV (cm/s) & $0.2 \pm 0.1$ & $1 \pm 0.3$ & $0.6 \pm 0.1$ & .0063 \\
RVCV (cm/s) & $22 \pm 2$ & $16 \pm 2$ & $17 \pm 1$ & .0272 \\
RVOT (ms) & $26 \pm 2$ & $33 \pm 3$ & $43 \pm 2$ & $<.0001$ \\
RVCT (ms) & $47 \pm 1$ & $61 \pm 4$ & $64 \pm 3$ & .004 \\
ET (ms) & $303 \pm 2$ & $325 \pm 8$ & $314 \pm 4$ & .065 \\
Vmax (cm/s) & $282 \pm 27$ & $220 \pm 32$ & $249 \pm 30$ & .356 \\
MVO (mm) & $8.5 \pm 0.8$ & $10.6 \pm 1.3$ & $10.2 \pm 0.3$ & .2171 \\
SVCD (mm) & $0.6 \pm 0.3$ & $2.1 \pm 0.5$ & $1.7 \pm 0.3$ & .0457 \\
Wall contact (\%) & 80 & 0 & 20 & .029
\end{tabular}

RVOV, Rapid valve-opening velocity; SVCV, slow valve-closing velocity; $R V C V$, rapid valve-closing velocity; $R V O T$, rapid valve-opening time; $R V C T$, rapid valve-closing time; $E T$, ejection time; $V \max$, maximum transaortic flow velocity; $M V O$, maximum valve opening; $S V C D$, slow valve-closing displacement.

outputs of 2 to $9 \mathrm{~L} / \mathrm{min}$ (Table 1 ). The RVOV and RVCV of the reimplanted valves (David) were significantly faster, and the corresponding rapid valve-opening and valve-closing times were significantly shorter than the corresponding parameters in remodeled valves (Yacoub) and control valves (Supra; RVOV: $69 \pm 10 \mathrm{~cm} / \mathrm{s}$ [David] vs $39 \pm 4 \mathrm{~cm} / \mathrm{s}$ [Yacoub] vs $42 \pm 4 \mathrm{~cm} / \mathrm{s}$ [Supra], $P=.0041 ; \mathrm{RVCV}: 22 \pm$ $2 \mathrm{~cm} / \mathrm{s}$ [David] vs $16 \pm 2 \mathrm{~cm} / \mathrm{s}$ [Yacoub] vs $17 \pm 1 \mathrm{~cm} / \mathrm{s}$ [Supra], $P=.0272$; rapid valve-opening time: $26 \pm 2 \mathrm{~ms}$ [David] vs $33 \pm 3 \mathrm{~ms}$ [Yacoub] vs $43 \pm 2 \mathrm{~ms}$ [Supra], $P<$ .0001 ; rapid valve-closing time: $47 \pm 1 \mathrm{~ms}$ [David] vs $61 \pm$ $4 \mathrm{~ms}$ [Yacoub] vs $64 \pm 3 \mathrm{~ms}$ [Supra], $P=.004$ ). By contrast, the SVCV was faster in the remodeled valves and the control valves $(0.2 \pm 0.1 \mathrm{~cm} / \mathrm{s}$ [David] vs $1.0 \pm 0.3$ $\mathrm{cm} / \mathrm{s}$ [Yacoub] vs $0.6 \pm 0.1 \mathrm{~cm} / \mathrm{s}$ [Supra], $P=.0063$ ). Maximum valve opening was smallest after reimplantation $(P=.2171)$, and corresponding to the low SVCV, cusp displacement during slow closing was smallest in these valves $(P=.0457)$. Despite the rapid opening and closing velocities of the reimplanted valves, ET was shorter in these valves $(P=.065)$, their maximum flow velocity was faster $(P=.356)$, and they showed significantly more frequent systolic wall contact with the aortic root $(P=.029)$. These differences were true for low and high cardiac output conditions (Tables 2 and 3).

Figure 3 shows typical M-mode recordings of aortic valves after reimplantation and remodeling. The more rectangular form of the aortic valve movement after reimplantation is caused by the faster opening and closing of the valve. By contrast, the remodeled valve displays smoother valve opening and closing and maintains its distance from the aortic wall; meanwhile, the reimplanted valve touches the aortic wall during the whole systole. 
TABLE 2. Valve-motion characteristics after reimplantation (David) and remodeling (Yacoub) and in control valves (Supra) at a cardiac output of $2 \mathrm{~L} / \mathrm{min}$

\begin{tabular}{lcccc}
\hline & David & Yacoub & Supra & $\boldsymbol{P}$ value \\
\hline RVOV (cm/s) & $49 \pm 8$ & $35 \pm 9$ & $29 \pm 6$ & .2110 \\
SVCV (cm/s) & $0.4 \pm 0.2$ & $1.1 \pm 0.6$ & $0.8 \pm 0.3$ & .4433 \\
RVCV (cm/s) & $23 \pm 5$ & $13 \pm 2$ & $14 \pm 1$ & .1235 \\
RVOT (ms) & $27 \pm 6$ & $41 \pm 4$ & $48 \pm 2$ & .0426 \\
RVCT (ms) & $49 \pm 2$ & $54 \pm 17$ & $72 \pm 10$ & .3338 \\
ET (ms) & $307 \pm 6$ & $333 \pm 28$ & $317 \pm 13$ & .4496 \\
Vmax (cm/s) & $130 \pm 10$ & $110 \pm 35$ & $105 \pm 14$ & .5732 \\
MVO (mm) & $10.4 \pm 0.7$ & $11.5 \pm 3.5$ & $9.3 \pm 0.3$ & .7989 \\
SVCD (mm) & $0.4 \pm 0.7$ & $2.8 \pm 1.8$ & $1.8 \pm 0.8$ & .4274 \\
Wall contact (\%) & 80 & 0 & 20 & .029 \\
\hline
\end{tabular}

RVOV, Rapid valve-opening velocity; SVCV, slow valve-closing velocity; $R V C V$, rapid valve-closing velocity; RVOT, rapid valve-opening time; $R V C T$, rapid valve-closing time; $E T$, ejection time; $V \max$, maximum transaortic flow velocity; $M V O$, maximum valve opening; $S V C D$, slow valve-closing displacement.

Compared with the remodeled valves and control valves, rapid opening velocity increased disproportionately with increasing cardiac output after reimplantation (Figure 4); meanwhile, RVCV after reimplantation remained almost unchanged (Figure 5). Reduction of the SVCV after increasing cardiac output appeared to be comparable in all 3 groups (Figure 6). The high standard error of the measured parameters indicates a remarkably high interindividual variability, despite the standardized anatomic and hemodynamic conditions.

\section{Discussion}

The influence of valve-sparing operations on valve-motion pattern has been described repeatedly in vivo. ${ }^{13-16}$ The

TABLE 3. Valve-motion characteristics after reimplantation (David) and remodeling (Yacoub) and in control valves (Supra) at a cardiac output of $9 \mathrm{~L} / \mathrm{min}$

\begin{tabular}{lcccc}
\hline & David & Yacoub & Supra & $\boldsymbol{P}$ value \\
\hline RVOV (cm/s) & $103 \pm 40$ & $43 \pm 7$ & $58 \pm 8$ & .2084 \\
SVCV (cm/s) & $0.1 \pm 0.1$ & $0.8 \pm 0.7$ & $0.5 \pm 0.2$ & .4521 \\
RVCV (cm/s) & $24 \pm 5$ & $20 \pm 4$ & $22 \pm 3$ & .7951 \\
RVOT (ms) & $25 \pm 3$ & $29 \pm 6$ & $39 \pm 2$ & .1671 \\
RVCT (ms) & $45 \pm 1$ & $64 \pm 7$ & $65 \pm 3$ & .0210 \\
ET (ms) & $299 \pm 1$ & $330 \pm 20$ & $316 \pm 4$ & .1758 \\
Vmax (cm/s) & $426 \pm 5$ & $337 \pm 35$ & $395 \pm 13$ & .0149 \\
MVO (mm) & $8.5 \pm 1.5$ & $11 \pm 1$ & $11.2 \pm 0.2$ & .2768 \\
SVCD (mm) & $0.5 \pm 0.5$ & $1.5 \pm 0.5$ & $1.9 \pm 0.6$ & .3045 \\
Wall contact (\%) & 80 & 0 & 20 & .029 \\
\hline
\end{tabular}

RVOV, Rapid valve-opening velocity; $S V C V$, slow valve-closing velocity; $R V C V$, rapid valve-closing velocity; $R V O T$, rapid valve-opening time; $R V C T$, rapid valve-closing time; $E T$, ejection time; $V \max$, maximum transaortic flow velocity; $M V O$, maximum valve opening; $S V C D$, slow valve-closing displacement.
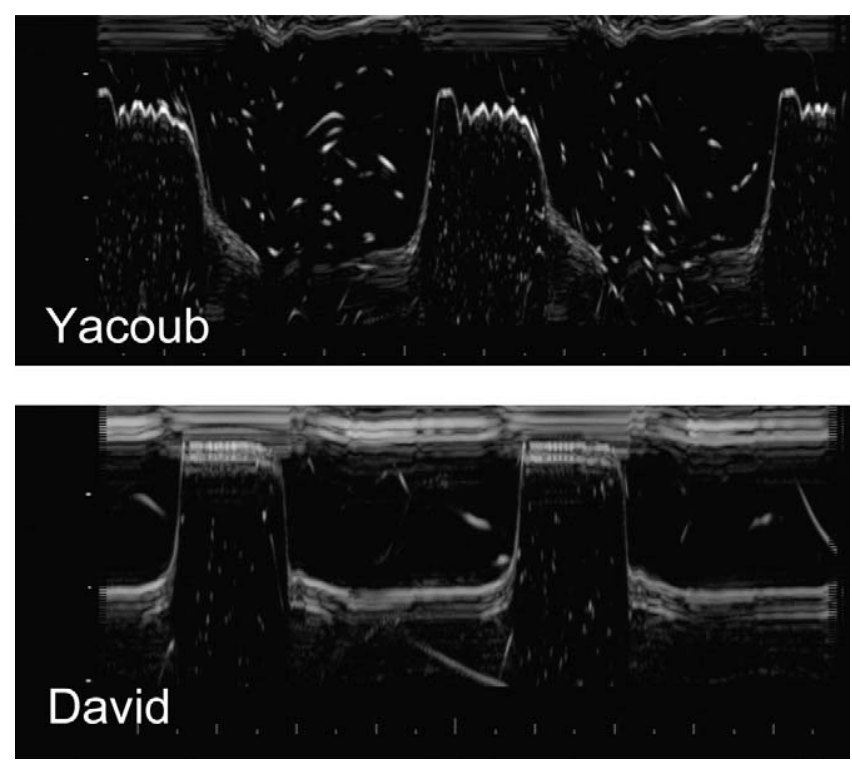

Figure 3. Typical M-mode recording of aortic valve motion after reimplantation (David) and remodeling (Yacoub).

results of these studies are limited by unstandardized hemodynamic conditions and variable echocardiographic windows determined by the individual anatomy of each patient. This study, for the first time, evaluates the opening and closing characteristics of the aortic valve after different modes of valve reconstruction under standardized hemodynamic and anatomic conditions in an experimental in vitro model simulating resting conditions and physical activity. We characterized systolic valve-opening and valve-closing movements during 3 distinct phases comprising rapid opening, slow closing, and rapid closing of the valve (Figure 2). Compared with reimplanted valves, we found significantly smoother valve movements in remodeled and control valves. Furthermore, we could substantiate the influences of cardiac output on valve motion, and we revealed a remarkably high dispersion of the measured parameters in spite of the standardized experimental setting.

In agreement with Leyh and associates, ${ }^{13}$ we found a smooth valve-motion pattern after remodeling of the aortic valve but not after reimplantation. We found that aortic cusps do open and close much more abruptly after reimplantation than after remodeling (Figure 3). This can be explained by the reduced distensibility of the aortic root after reimplantation, ${ }^{13,15}$ which increases dynamic pressure load onto the aortic cusps. Increased rapid opening and closing velocities were accompanied by a decreased slow closing movement of the reimplanted valves (Tables 1-3 and Figure 6). This slow closing happens physiologically during decreasing forward flow across the aortic valve, diminishes the leaflet excursion during rapid valve closing, 


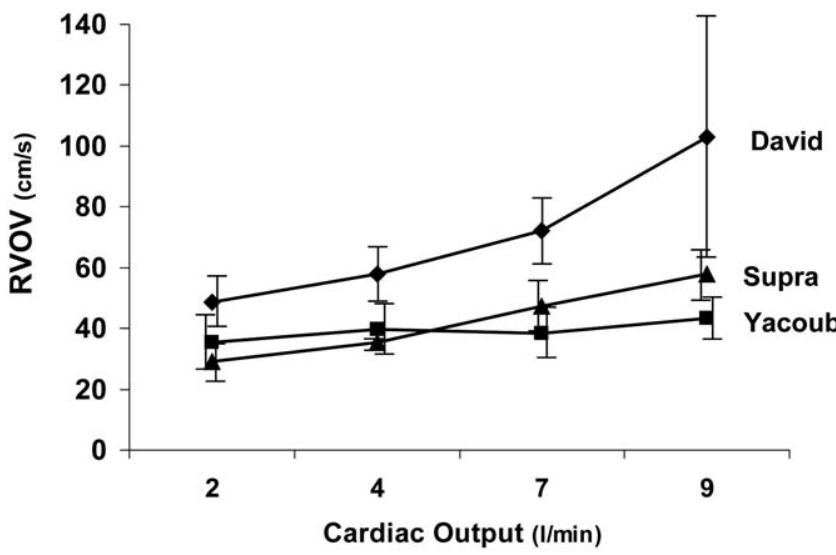

Figure 4. Rapid valve-opening velocity (RVOV) after reimplantation (David) and remodeling (Yacoub) and in control valves (Supra).

and thus minimizes closing stress. The lack of sinuses after valve reimplantation can explain the reduced slow closing movement because it has been demonstrated that the eddy currents inside the sinuses initiate closing of the valve already during ejection. The reduction in slow valve closing, however, also corresponds to a smaller maximum opening of reimplanted valves and a trend toward reduced ET (Tables 1-3): if a valve has less total opening time and less maximum opening displacement, forward flow will decrease less during late systole to eject a defined stroke volume. This might also help to explain why slow valveclosing displacement was reduced and maximum flow velocity was increased in reimplanted valves (Tables 1-3). Limited valve opening after reimplantation was also reflected by the frequent systolic wall contact of the reimplanted valves (Tables 1-3), which might again increase dynamic stress on the valve cusps. Furthermore, the lack of sinuses after reimplantation prevents the blood stream from forming vortices between the aortic cusps and the aortic wall, which might be important for positioning the aortic leaflets without contact with the aortic wall. ${ }^{20}$

Our experimental setting allowed a detailed analysis of how cardiac output influences valve motion. Rapid opening velocity increased disproportionately with increasing cardiac output in reimplanted valves (Figure 4), whereas rapid valve closing remained almost unchanged after reimplantation (Figure 5). The decrease in SVCV after increasing cardiac output was comparable in all 3 groups of valves (Figure 6). Thus the difference between reimplanted valves and those after remodeling or control valves increased with respect to rapid valve opening, remained unchanged with respect to slow valve closing, and decreased with respect to rapid valve closing. Considering all 3 movement phases as equally important, higher cardiac output does not seem to increase the unphysiologic character of valve motion after

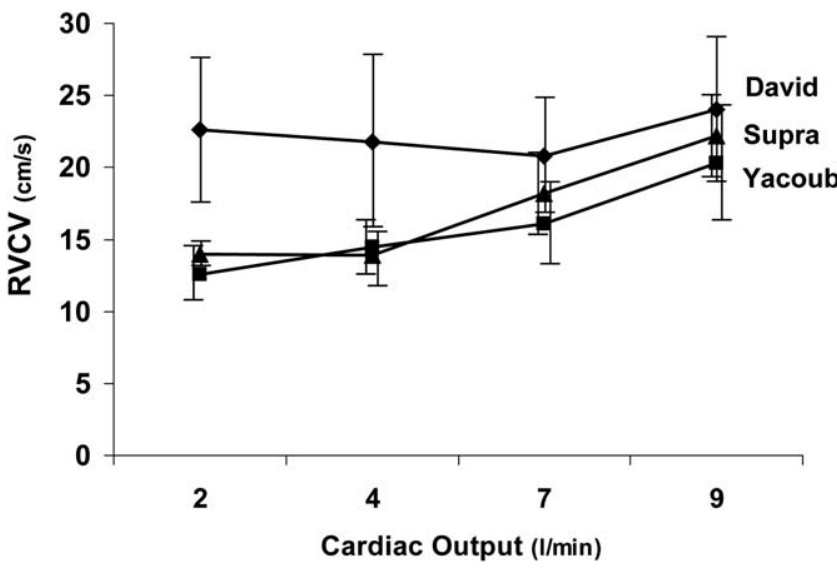

Figure 5. Rapid valve-closing velocity (RVCV) after reimplantation (David) and remodeling (Yacoub) and in control valves (Supra).

reimplantation in comparison with remodeled or control valves. It can be speculated that this in part explains why the reimplantation technique shows a comparable rate of late failure during midterm follow-up in spite of the smoother valve motions after remodeling. ${ }^{5-8}$

Another important finding of our series is the considerably high variability of the measured parameters despite the standardized experimental conditions. This can possibly be explained by minimal yet unapparent differences in surgical technique, unappreciated differences in properties of the porcine valves, and bending deformation induced by the operation. It can be aggravated by the M-mode technique, which represents a 1-dimensional method. However, this observation in a standardized in vitro experiment and the demonstrated influence of cardiac output on valve motion suggest that measurements during unstandardized in vivo conditions should be interpreted cautiously.

Our results contradict the results of Leyh and associates ${ }^{13}$ with respect to rapid closing velocity and ET. Rapid closing velocity in our series was increased and not decreased and ET was shortened and not prolonged in reimplanted valves. In our opinion a higher RVCV and a shortened ET is more consistent to the valve-motion pattern after reimplantation. It has been shown that semilunar valves start to open before any forward flow because of root expansion during the beginning of systole. ${ }^{12,21}$ This early opening of the valve is M-mode echocardiographically recorded as part of ejection, despite the lack of forward flow. After reimplantation, root expansion is practically impossible, ${ }^{13}$ preventing the valve from early opening and thus shortening the measured ET. In conjunction with a defined stroke volume, a shorter opening time will require abrupt valve motion. Furthermore, the reduced slow closing of the reimplanted valves increases the necessary valve displacement during rapid closing, and this should increase velocity dur- 


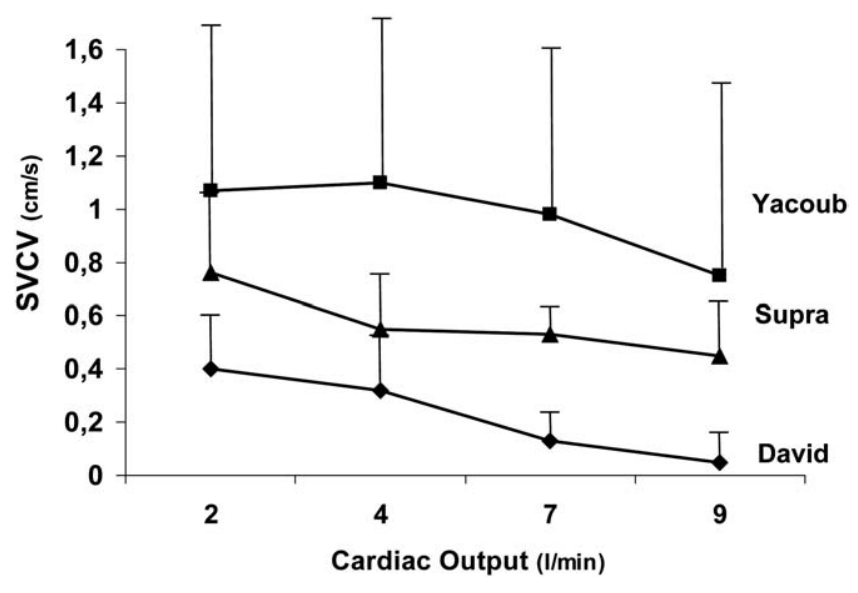

Figure 6. Slow valve-closing velocity (SVCV) after reimplantation (David) and remodeling (Yacoub) and in control valves (Supra).

ing rapid valve closing. The obvious less favorable valve motion after the David I procedure has led to the development of more than 5 variations of the original David technique described in the last few years. ${ }^{22-24}$

The statistical significance of our results might be limited by the relatively small number of 5 valves in each group. This aspect only emphasizes the apparent differences between the groups. The results might further be influenced by the fact that saline has a different viscosity compared with blood. Nevertheless, testing conditions were identical for all 3 groups.

We conclude that remodeling of the aortic valve provides a smoother valve mobility, and meanwhile, reimplantation of the aortic valve after the David I procedure results in significantly faster valve opening and closing with frequent systolic valve contact with the aortic wall. To date, it remains unclear whether the softer valve-motion pattern after remodeling might translate into superior valve performance during long-term follow-up.

\section{References}

1. Sarsam MA, Yacoub M. Remodeling of the aortic annulus. $J$ Thorac Cardiovasc Surg. 1993;105:435-8.

2. David TE, Feindel CF. An aortic valve sparing operation for patients with aortic incompetence and aneurysm of the ascending aorta. J Thorac Cardiovasc Surg. 1992;103:617-22.

3. David TE. Remodeling of the aortic root and preservation of the native aortic valve. Card Thorac Surg. 1996;1:44-56.
4. Schäfers H-J, Fries R, Langer F, Nikolaudakis N, Graeter T, Grundmann $\mathrm{U}$. Valve preserving replacement of the ascending aorta: remodeling versus reimplantation. J Thorac Cardiovasc Surg. 1998;116:990-6.

5. Yacoub MH, Gehle P, Chandrasekaran V, Birks EJ, Child A, RadleySmith R. Late results of a valve-preserving operation in patients with aneurysms of the ascending aorta and root. J Thorac Cardiovasc Surg. 1998;115:1080-90.

6. David TE, Armstrong S, Ivanov J, Feindel CM, Omran A, Webb G. Results of aortic valve-sparing operations. J Thorac Cardiovasc Surg. 2001;122:39-46.

7. Miller C. Discussion: session 3-dissection. Ann Thorac Surg. 2002; 74(suppl):S1857-63.

8. Graeter T, Aicher D, Langer F, Wendler O, Schäfers H-J. Midterm results of aortic valve preservation: remodeling vs. reimplantation. Thorac Cardiovasc Surg. 2002;50:21-4.

9. Brewer RJ, Deck JD, Capati B, Nolan SP. The dynamic aortic root: its role in aortic valve function. $J$ Thorac Cardiovasc Surg. 1976;72:413-7.

10. Gnyaneshwar R, Kumar RK, Balakrishnan KR. Dynamic analysis of the aortic valve using a finite element model. Ann Thorac Surg. 2002;73:1122-9.

11. Handke M, Heinrichs G, Beyersdorf F, Olschewski M, Bode C, Geibel A. In vivo analysis of aortic valve dynamics by transesophageal 3 -dimensional echocardiography with high temporal resolution. $J$ Thorac Cardiovasc Surg. 2003;125:1412-9.

12. Higashidate M, Tamiya K, Beppu T, Imai Y. Regulation of the aortic valve opening. In vivo dynamic measurement of aortic valve orifice area. J Thorac Cardiovasc Surg. 1995;110:496-503.

13. Leyh RG, Schmidtke C, Sievers HH, Yacoub MH. Opening and closing characteristics of the aortic valve after different types of valve-preserving surgery. Circulation. 1999;100:2153-60.

14. De Paulis R, De Matteis GM, Nardi P, Scaffa R, Buratta MM, Chiariello L. Opening and closing characteristics of the aortic valve after valve-sparing procedures using a new aortic root conduit. Ann Thorac Surg. 2001;72:487-94.

15. De Paulis R, De Matteis GM, Nardi P, Scaffa R, Bassano C, Chiariello L. Analysis of valve motion after the reimplantation type of valvesparing procedure (David I) with a new aortic root conduit. Ann Thorac Surg. 2002;74:53-7.

16. Aybek T, Sotiriou M, Wohleke T, Miskovic A, Simon A, Doss M, et al. Valve opening and closing dynamics after different aortic valvesparing operations. J Heart Valve Dis. 2005;14:114-20.

17. Reul H, van Son JA, Steinseifer U, Schmitz B, Schmidt A, Schmitz C, et al. In vitro comparison of bileaflet aortic heart valve prostheses. J Thorac Cardiovasc Surg. 1993;106:412-20.

18. Knott E, Reul H, Knoch M, Steinseifer U, Rau G. In vitro comparison of aortic heart valve prostheses. I. Mechanical valves. J Thorac Cardiovasc Surg. 1988;96:952-61.

19. Food and drug administration test protocol: interlaboratory comparison of prosthetic heart valve performance testing. Rockville (MD): Food and Drug Administration; 1985.

20. Bellhouse BJ, Bellhouse FH. Mechanism of closure of the aortic valve. Nature. 1968;217:86-7.

21. Thubricar MJ, Heckmann JL, Nolan SP. High speed cine-radiographic study of aortic valve leaflet motion. J Heart Valve Dis. 1993;26: 653-61.

22. Miller DC. Valve-sparing aortic root replacement in patients with the Marfan syndrome. J Thorac Cardiovasc Surg. 2003;125:773-8.

23. Gleason TG. New graft formulation and modification of the David reimplantation technique. J Thorac Cardiovasc Surg. 2005;130:601-3.

24. Demers P, Miller DC. Simple modification of "T. David-V" valvesparing aortic root replacement to create graft pseudosinuses. Ann Thorac Surg. 2004;78:1479-81. 